\title{
Diagnostic Value of Ultrasound-Guided Pleural Biopsy in Patients with Recurrent Pleural Effusion
}

Mohamed Azzam ${ }^{1}$, Tarek Mohsen ${ }^{1}$, Sally Fouad Tadros ${ }^{2}$, Hesham Zayed Saleh ${ }^{1}$

Departments of ${ }^{1}$ Cardiothoracic Surgery and ${ }^{2}$ Radiology, Kasr Al Ainy Faculty of Medicine, Cairo University, Egypt

*Corresponding author: Mohamed Azzam, E-mail: dr.mohamed.ezzazzam@gmail.com

\begin{abstract}
Background: Establishing a definite diagnosis in cases of pleural effusion is sometimes challenging. Adding ultrasound-guided tru-cut biopsy at the time of obtaining pleural fluid for analysis may increase the diagnostic yield in such cases.

Objective: The aim of the current work was to assess the diagnostic yield and the safety of percutaneous ultrasound guided tru-cut needle biopsy in patients with recurrent undiagnosed pleural effusion.

Patients and Methods: A retrospective observational study including 45 patients with recurrent undiagnosed pleural effusion who underwent ultrasound-guided tru-cut needle biopsy in the period between January 2019 and October 2020. Results: By using ultrasound-guided needle biopsy, we were able to reach a final histopathological diagnosis in 40 patients with a diagnostic yield of $88.8 \%$. Only 5 cases remained undiagnosed (11.1\%). Mesothelioma was found in 13 cases $(28.9 \%)$ and bronchogenic carcinoma in 7 cases (15.5\%). Post-procedural complications included 1 case of pneumothorax (2.2\%) and 1 case of hemothorax (2.2\%) all resolving with the small caliber chest drain that was inserted at the conclusion of the procedure.

Conclusion: It could be concluded that ultrasound-guided needle biopsy is a safe procedure that can be useful in establishing a diagnosis in patients with recurrent pleural effusion. Its high diagnostic yield, while being simpler and cheaper than CT guided biopsy or thoracoscopy, makes it a suitable option in our clinical settings. More studies are needed to validate our findings.
\end{abstract}

Keywords: Pleural effusion, Tru-cut needle biopsy, Ultrasound

\section{INTRODUCTION}

Establishing a diagnosis in cases of pleural effusion is sometimes difficult. In many cases no definite diagnosis is reached even after complete investigations ${ }^{(\mathbf{1})}$. Obtaining pleural tissue, especially in the presence of pleural thickening or masses may help establishing a diagnosis. The classical alternatives usually used to obtain biopsy material are either CTguided biopsy or thoracoscopy $(\mathbf{1}, \mathbf{2}, \mathbf{3})$.

Over the past years, ultrasound assessment has gained grounds as a useful bedside tool guiding thoracocentesis and placement of chest drains (4). Moreover, Ultrasound can also delineate pleural masses as well as lung tumors involving or abutting the pleura $(5,6$ ). Extending the use of ultrasound to guide cuttingneedle biopsies from such lesions would offer many potential advantages over CT-guided techniques or thoracoscopy. With the lighter more mobile modern ultrasound units, such procedures can be arranged and performed in a much easier and faster way by the pulmonologist or thoracic surgeon. Advantages over CT guidance also include real-time imaging allowing compensation for breathing motion thus increasing the safety margin of the procedure while minimizing patient's exposure to ionizing radiation. Previous studies comparing the ultrasound to CT-guidance showed ultrasound to be at least equivalent to $\mathrm{CT}$ for needle biopsies of suitable chest lesions ${ }^{(6-8)}$. Other authors found ultrasound guidance to result in a higher likelihood of a diagnostic sample ${ }^{(9)}$. Such advantages make ultrasound-guided biopsies more suitable for our clinical settings.

The objective of the current study was to assess the diagnostic yield and the safety of percutaneous ultrasound-guided needle biopsy in patients with recurrent pleural effusion.

\section{PATIENTS AND METHODS}

This retrospective observational study included a total of 45 successive patients presented with recurrent pleural effusion as evidenced by recent radiologic examination, attending at Cardiothoracic Surgery Department of Cairo University Hospitals. This study was conducted between January 2019 and October 2020.

During the study period 48 consecutive patients remained undiagnosed despite previous pleural fluid chemical and cytological examination. Three patients were excluded; one of them due to features of pleural aspirate compatible with empyema, one due to transudative pattern on previous pleural aspirate and the last one was excluded due to severe emphysema. The remaining 45 patients were included in the study.

All patients underwent CT chest with contrast prior to the procedure to gain proper delineation of the target lesions if any and the surrounding anatomy. Platelet counts, international normalized ratio (INR) and activated partial thromboplastin time (aPTT) were obtained to ensure the absence of coagulopathy prior to the procedure.

\section{Ethical Consideration:}


The study was approved by the local ethical committee of Cairo University. Written consent was obtained from all patients prior to the procedures. This work has been carried out in accordance with The Code of Ethics of the World Medical Association (Declaration of Helsinki) for studies involving humans.

\section{Procedure:}

Patients were monitored with ECG, noninvasive blood pressure cuffs, and oximetry for the procedure. Procedure started with a complete ultrasound scan of the hemithorax in question. This was done using the HD 11 XE machines (Phillips Healthcare, Andover, MA) with curvilinear transducer probe $(\mathrm{C} 5-2 \mathrm{MHz})$, phased array probe $(3.5 \mathrm{MHz})$ and linear array transducer probe $(\mathrm{L} 12-4 \mathrm{MHz})$, in the Diagnostic interventional OR Unit in the Cardiothoracic Department of Cairo University Hospitals.

After determining the best biopsy site, the skin was scrubbed with iodopovidone, and after anesthetizing the skin up to the parietal pleura using 10 $\mathrm{ml}$ of $2 \%$ lidocaine, a $0.5 \mathrm{~cm}$ transverse skin incision was made by a scalpel parallel to the upper border of the rib. The direction and depth of the biopsy needle trajectory were determined by ultrasound. Color-coded Doppler imaging was used to identify surrounding vessels in cases of doubt. Core biopsies of the pleura were performed using tru-cut needle (Gauge 18) with a specimen notch of $20 \mathrm{~mm}$, at least 3-4 cores were taken, fixed in formaldehyde solution, and transferred for histopathological examination.

A pigtail $12 \mathrm{~F}$ chest drain was inserted at the conclusion of the procedure under sonar guidance to follow-up drainage and for chemical pleurodesis later if indicated. Patients were monitored for two hours postprocedure and a plain chest $\mathrm{x}$-ray was obtained 3-4 hours later to exclude any collection or pneumothorax.

Relevant data of the 45 patients were collected from individual patient files. Continuous variables were expressed as mean \pm standard deviation (SD). Categorical variables were expressed as numbers and percentages.

\section{Statistical analysis}

The collected data were coded, processed and analyzed using the SPSS (Statistical Package for Social Sciences) version 22 for Windows ${ }^{\circledR}$ (IBM SPSS Inc, Chicago, IL, USA).

\section{RESULTS}

Table 1 shows the patients' characteristics with a mean age of $53.1 \pm 11.1$ years with a male majority (64.4\%). 60\% of the patients had a smoking history and the majority had one previous thoracocentesis. $13.4 \%$ had more than one prior thoracocentesis that was insufficient to provide a diagnosis. In 23 patients the effusion was right sided and in three patients the effusion was bilateral. A pleural mass was identifiable only in $17.8 \%$ of the cases and pleural thickening more than $5 \mathrm{~mm}$ was present in the majority of the cases (66.7\%) (Table. 2).

Table 3 outlines the final pathological diagnosis. The most common diagnosis was malignant mesothelioma in $28.9 \%$ of the cases followed by bronchogenic carcinoma in $15.5 \%$ of the cases. In only 5 cases no histopathological diagnosis was established; with three of these showing essentially normal pleural tissue, and two cases with non-specific inconclusive findings.

There were only one incidence of pneumothorax with the development of mild surgical emphysema and one case of hemothorax. Both resolved with the small caliber chest drain that was routinely inserted at the conclusion of the procedure to drain the pleural effusion.

Table (1): Patient characteristics.

\begin{tabular}{|l|c|}
\hline & $\mathbf{N}(\%)$ \\
\hline Age (years) (Mean \pm SD) & $53.1 \pm 11.1$ \\
\hline Sex & $29(64.4 \%)$ \\
Male & $16(35.6 \%)$ \\
Female & $27(60 \%)$ \\
\hline Smoking History & $10(22.2 \%)$ \\
\hline Occupational hazard & $39(86.6 \%)$ \\
\hline Single previous Thoracocentesis & $6(13.4 \%)$ \\
\hline $\begin{array}{l}\text { More than one previous } \\
\text { thoracocentesis }\end{array}$ \\
\hline
\end{tabular}

Table (2): Ultrasound finding.

\begin{tabular}{|c|c|c|}
\hline \multicolumn{2}{|c|}{ (2) } & $\mathbf{N}(\%)$ \\
\hline \multirow{3}{*}{$\begin{array}{ll}\text { Side } & \text { of } \\
\text { effusion } & \end{array}$} & Isolated RT. & $23 / 45(51.1$ \\
\hline & Isol & $19 \backslash 45$ \\
\hline & Bil & $3 \backslash 4$ \\
\hline \multirow{2}{*}{$\begin{array}{l}\text { Side of tru- } \\
\text { cut biopsy }\end{array}$} & R & $26 / 4$ \\
\hline & & $19 / 4$ \\
\hline \multirow{2}{*}{$\begin{array}{l}\text { Pleural } \\
\text { thickness }\end{array}$} & Less $\mathrm{t}$ & $7 / 45(15.6 \%)$ \\
\hline & Mor & $38 / 45(84.4 \%)$ \\
\hline \multirow[t]{2}{*}{ Pleural mass } & Yes & $8 / 45(17.8 \%)$ \\
\hline & No & $37 \backslash 45(82.2 \%)$ \\
\hline
\end{tabular}

Table (3): Biopsy results.

\begin{tabular}{|l|c|}
\hline \multicolumn{1}{|c|}{ Diagnosis } & $\mathbf{N}(\mathbf{\%})$ \\
\hline Malignant Mesothelioma & $13(28.9 \%)$ \\
\hline Non small-cell lung cancer & $5(11.1 \%)$ \\
\hline Small-cell lung cancer & $2(4.4 \%)$ \\
\hline $\begin{array}{l}\text { Metastatic form extra- } \\
\text { pulmonary primary }\end{array}$ & $4(8.8 \%)$ \\
\hline Lymphoma & $1(2.2 \%)$ \\
\hline Benign pleural tumor & $2(4.4 \%)$ \\
\hline Tuberculous granuloma & $3(6.6 \%)$ \\
\hline Pleural fibrosis \& plaques & $7(15.5 \%)$ \\
\hline Inflammatory pleuritis & $3(6.6 \%)$ \\
\hline Undiagnosed & $5(11.1 \%)$ \\
\hline
\end{tabular}

\section{DISCUSSION}

Reaching a definite diagnosis in cases presenting with pleural effusion is sometimes a difficult task 
especially in the case of malignancy. Using a panel of tumor markers was previously reported but proved to lack specificity ${ }^{\left({ }^{10}\right)}$. Cytological analysis of pleural fluid remains the initial diagnostic test, but even recent publications report a relatively low diagnostic yield ranging around $46-67 \%$, with significant variability depending on the type of malignancy ${ }^{(11,12)}$.

Especially in the case of malignant mesothelioma, the diagnostic yield of cytological studies is known to be low (11-13). This may explain at least in part, the inability to reach a diagnosis in our group of patients on prior cytological examinations. Malignant mesothelioma was the commonest diagnosis in almost one third of our patients (28.9\%). Such findings are not surprising as many authors documented a higher and rising incidence of malignant mesothelioma in Egypt when compared to other regions of the world ${ }^{(14,15)}$. The second commonest diagnosis in our group of patients was bronchogenic carcinoma (15.5\%).

Although in such cases the diagnostic yield of cytological analysis is higher than mesothelioma and is reported to be highest in cases of adenocarcinoma ${ }^{(11,12)}$, it still remains far from perfect and further diagnostic workup is needed in many cases. In such undiagnosed cases the definitive diagnosis relies on histopathological examination of a pleural biopsy. Currently this is usually obtained through CT guidance or thoracoscopy. Since thoracoscopy is a more invasive \& more expensive modality, CT-guided biopsy is often contemplated first.

The diagnostic sensitivity for malignancy with CT-guided cutting-needle biopsy was previously reported to be around $87 \%{ }^{(2)}$. More recently, Niu et al. (16) reported almost similar diagnostic accuracy (89.2\%) in a patient series with pleural lesions not limited to malignancy. However their study reflected a commonly encountered practice, where patients with a pleural lesion thickness of less than $5 \mathrm{~mm}$ were excluded. Despite including such patients in the current study, the diagnostic yield was almost similar (88.8\%) using ultrasound guidance.

Over the past decade, thoracic ultrasound proved to be a valuable addition to the armamentarium of pulmonologists and thoracic surgeons and its applications have extended from being used just to guide drainage of pleural effusion into guiding needle biopsies from pleural, peripheral pulmonary and chest wall lesions ${ }^{(1,6,7)}$. Diacon et al. ${ }^{(17)}$ demonstrated a sensitivity of $85.5 \%$ for malignancy using ultrasoundguided needle biopsy in the hands of pulmonologists with an incidence of $4 \%$ pneumothorax. Other studies comparing ultrasound-guided to CT-guided biopsies showed comparable results ${ }^{(7,8)}$.

Sconfienza et al. ${ }^{(7)}$ concluded that ultrasoundguidance was comparable to CT in terms of sample accuracy while reducing procedure time, radiation exposure and incidence of pneumothorax. Jarmakani et al. ${ }^{(9)}$ found ultrasound guidance to be associated with a higher likelihood of a diagnostic sample in patients with peripheral intrathoracic and chest wall lesions. Our results with a diagnostic yield of $88.8 \%$ and a pneumothorax incidence of $2.2 \%$ are comparable to the results of all these previously mentioned studies, although many of them limited the use of ultrasound guidance to cases with more sizeable lesions ${ }^{(9,17)}$.

Previous studies from Egypt also reported results comparable to ours. Although their study reported a smaller number of patients (30 patients) and was limited to pleural lesions more than $20 \mathrm{~mm}$ in diameter, Bahr et al. ${ }^{(18)}$ reported a diagnostic yield of $80 \%$ with $30 \%$ of the cases being malignant mesothelioma. The current study thus supports a growing body of evidence documenting a satisfactory diagnostic yield of ultrasound-guided needle biopsy, which is at least comparable to CT guidance, but with the advantage of being a faster, cheaper and sometimes safer option.

When comparing the performance of ultrasoundguided biopsy to thoracoscopy, Sobhy et al. ${ }^{(19)}$ found thoracoscopy to have a higher sensitivity (94\% vs $77.7 \%$ ) in diagnosing pleural diseases. On the contrary Zhou et al. (20) found both modalities to have statistically similar diagnostic yields in cases of pleural effusion after inconclusive thoracocentesis. With such findings ultrasound-guided biopsy being a simple cheap procedure would be favored to thoracoscopy, a more expensive \& invasive procedure that is not necessarily available in small peripheral units in the country.

The limitations of our study include a limited number of patients, which were reviewed in a retrospective fashion. The fact all the patients are from one hospital and were all undiagnosed after at least one prior thoracocentesis may have introduced some form of a sampling bias, which limits the generalizability of our findings.

\section{CONCLUSION}

It could be concluded that ultrasound-guided needle biopsy is a safe procedure that can be useful in establishing a diagnosis in patients with recurrent pleural effusion. Its high diagnostic yield, while being a simpler and cheaper option than other modalities, makes it a suitable diagnostic alternative in our clinical settings. More studies are needed to validate our findings.

\section{REFERENCES}

1. Hooper C, Lee $\mathrm{Y}$, Maskell $\mathrm{N}$ et al. (2010): Investigation of a unilateral pleural effusion in adults: British Throacic Society pleural disease guideline 2010. Thorax, 65(2): 4-17.

2. Maskell N, Gleeson F, Davies R (2003): Standard pleural biopsy versus CT-guided cutting needle biopsy for diagnosis of malignant disease in pleural effusion: A randomized controlled trial. Lancet, 361:1326-31.

3. Hansen M, Faurschou P, Clementsen $P$ (1998): Medical thoracoscopy, results and complications in 146 patients: a retrospective study. Respir Med., 92: 228-32.

4. Diacon A, Brutsche M, Soler M (2003): Accuracy of pleural puncture sites. A prospective comparison of 
clinical examination with ultrasound. Chest, 123:43641.

5. Beckh S, Bolcskei P, Lessnau K (2002): Real-time chest ultrasonography: a comprehensive review for the pulmonologist. Chest, 122:1759-73.

6. Herth F, Becker H (2003): Transthoracic ultrasound. Respiration, 70:87-94.

7. Sheth S, Hamper U, Stanley D et al. (1999): US guidance for thoracic biopsy: a valuable alternative to CT. Radiology, 210:721-26.

8. Sconfienza L, Mauri G, Grossi F et al. (2013): Pleural and peripheral lung lesions: comparison of US and CTguided biopsy. Radiology, 266:930-35.

9. Jarmakani M, Duguay S, Rust K et al. (2016): Ultrasound versus computed tomographic guidance for percutaneous biopsy of chest lesions. J Ultrasound Med., 35:1865-72.

10. Porcel J, Vives M, Esquerda A et al. (2004): use of a panel of tumor markers (carcinoembryonic antigen, cancer antigen 125, carbohydrate antigen 15-3, and cytokeratin 19 fragments) in pleural fluid for the differential diagnosis of benign and malignant effusions. Chest, 126:1757-63.

11. Loveland $P$, Christie $M$, Hammerschlag $G$ et al. (2018): Diagnostic yield of pleural fluid cytology in malignant effusions: an Australian tertiary centre experience. Int Med J., 48(11): 1318-24.

12. Arnold D, De Fonseka D, Perry A et al. (2018): Investigating unilateral pleural effusions: the role of cytology. Eur Repir J., 52(5): 180-4.

13. Rakha E, Patil S, Abdulla $K$ et al. (2010): The sensitivity of cytologic evaluation of pleural fluid in the diagnosis of malignant mesothelioma. Dagn Cytopathol., 38:874-79.

14. Gaafar R, Aly Eldin N (2005): Epidemic of mesothelioma in Egypt. Lung Cancer, 49: 17-20.

15. Aly Eldin N, Mourad I (2003): Epidemiology of malignant pleural mesothelioma, 11 years experience at NCI-Cairo University, Poster No 3 in the 2nd NCIEORTC Lung Cancer Symposium, Sharm-Elsheikh, Egypt, Pp. 26-28.

16. Niu X, Bhetuwal A, Yang H (2015): CT-guided core needle biopsy of pleural lesions: evaluating diagnostic yield and associated complications. Korean J Radiol., 16: 206-12.

17. Diacon A, Schuurmans M, Theron J et al. (2004): Safety and yield of ultrasound-assisted transthoracic biopsy performed by pulmonologist. Respiration, 71:519-22.

18. Bahr H, El-Shafey M, Hantera M et al. (2013): Ultrasound guided needle pleural biopsy in patients with undiagnosed pleural effusion. Egyptian J Chest Diseas \& Tuberculosis, 63: 113-18.

19. Sobhy K, Kamal K, Ahmed S et al. (2017): Ultrasound-guided closed pleural biopsy versus medical thoracoscopic pleural biopsy in diagnosis of pleural diseases. Egyptian J Chest Diseas \& Tuberculosis, 66 (1): 97-106.

20. Zhou X, Jiang $P$, Huan $X$ et al. (2018): UltrasoundGuided versus Thoracoscopic Pleural Biopsy for Diagnosing Tuberculous Pleurisy Following Inconclusive Thoracentesis: A Randomized, Controlled Trial. Med Sci Monit., 24: 7238- 48. 